\title{
PREDICTORES DE AUTOEFICACIA PARA EL CONTROL DE PESO EN ESTUDIANTES ADOLESCENTES
}

\author{
PREDICTORS OF SELF-EFFICACY FOR WEIGHT CONTROL IN ADOLESCENT STUDENTS
}

León Hernández Rodrigo C.*, Méndez Santos Gregorio**,

Torres-Compeán Yulissa Guadalupe**, Gilda Gómez-Peresmitré.***

Consejo Nacional de Ciencia y Tecnología *, Universidad Autónoma de Tamaulipas**,

Universidad Nacional Autónoma de México.***

Correspondencia: rcleonhe@conacyt.mx

\section{RESUMEN}

Así como existen factores que conllevan un riesgo en las personas que presentan problemas para el control de peso, se encuentran los que promueven cambios favorables en las conductas de salud, entre ellos la autoeficacia se le ha considerado como un factor de gran importancia en el mantenimiento de la salud, ya que contribuye en la mejora de los hábitos e influye directamente en la percepción del bienestar y la actitud positiva que se tiene ante el cambio de conductas saludables. El objetivo del presente estudio fue identificar predictores de autoeficacia para el control de peso en estudiantes de secundaria (públicas y privadas) de la zona sur de Tamaulipas (Tampico y Ciudad Madero). El diseño del estudio fue no experimental, explicativo y transversal. La muestra fue no probabilística e intencional de 343 adolescentes estudiantes de escuelas secundarias públicas y privadas. 
Se utilizó el inventario de Autoeficacia Percibida para el Control de Peso de estudiantes de secundaria, el índice de masa corporal (IMC), la escala para evaluar aspectos positivos y negativos (PANAS) y el inventario de Autoatribuciones positivas y negativas en adolescentes. La edad promedio de los adolescentes de este estudio fue de 13.48, el 79\%. Los resultados muestran una relación lineal significativa entre la variable dependiente y dos variables independientes $(F=52.83, p<.001)$, autoatributos positivos es la que mejor predice autoeficacia para el control de peso $(\beta=.38)$, seguida por afectos positivos $(\beta=.15)$. Se puede concluir que el modelo predictivo de autoeficacia para el control de peso en estudiantes adolescentes de la zona sur de Tamaulipas es significativo y cumple con el objetivo de este estudio.

Palabras clave: Autoeficacia, control de peso, autoatributos, afectos, estudiantes

\section{ABSTRACT}

Just as there are factors that carry a risk in people who have weight control problems, there are those that promote favorable changes in health behaviors, among them, self-efficacy has been considered as a factor of great importance in maintaining health, since it contributes to the improvement of habits and directly influences the perception of wellbeing and the positive attitude that one has when faced with the change of healthy behaviors. The objective of this study was to identify predictors of self-efficacy for weight control in junior high school students (public and private) from the southern part of Tamaulipas (Tampico and Ciudad Madero). The study design was non-experimental, explanatory, and cross-sectional. The sample was non-probabilistic and intentional of 343 adolescent students from public and private junior high schools. 
The inventory of Perceived Self-efficacy for Weight Control of junior high school students, the body mass index (BMI), the scale to evaluate positive and negative aspects (PANAS) and the inventory of positive and negative self-attributions in adolescents were used. The average age of the adolescents in this study was $13.48,79 \%$. The results show a significant linear relationship between the dependent variable and two independent variables $(F=52.83, p<.001)$, Positive self-attributes is the one that best predicts self-efficacy for weight control $(\beta=.38)$, followed by positive affects $(\beta=.15)$. It can be concluded that the predictive model of selfefficacy for weight control in adolescent students in the southern area of Tamaulipas is significant and meets the objective of this study.

Key words: Self-efficacy, weight control, self attributes, affections, students.

Esta investigación fue subvencionada por el Programa de Apoyo a Proyectos de Investigación e Innovación Tecnológica (PAPIIT-UNAM), Proyecto IN310620

\section{INTRODUCCIÓN}

Hoy en día la malnutrición es uno de los problemas sanitarios con mayor afectación a nivel mundial (Organización Mundial de la Salud [OMS] 2018). Uno de estos problemas es la desnutrición, principalmente ocasionada por la deficiente calidad y cantidad de alimentos consumidos (Gómez, 2016). En el otro extremo se ubica la obesidad y el sobrepeso, que se caracteriza por incremento desmesurado de peso corporal que suele iniciar en la infancia y adolescencia (Dávila-Torres, González-Izquierdo \& Barrera-Cruz, 2015) y cuyas causas se asocian a factores biológicos, ambientales y culturales (Gutiérrez, et al. 2017). 
Durante el 2016, en el mundo existían más de 1900 millones de personas de 18 años o más con sobrepeso u obesidad y aproximadamente 41 millones de niños menores de 5 años presentaban esta problemática (OMS, 2020). En México, la Encuesta Nacional de Salud y Nutrición del 2018 detectó que en poblaciones de 5 a 11 y de 12 a 19 años, los porcentajes de obesidad fueron de $17.5 \%$ y $14.1 \%$ respectivamente. Los porcentajes más altos de obesidad en poblaciones de 12 a 19 años se ubicaron en el estado de Veracruz (22.9\%), Quintana Roo (22.8\%), Colima (24.9\%), Sonora (22.2\%) y Tabasco (19.9\%). En Tamaulipas se detectó un 16.9\% ("Encuesta Nacional de Salud y Nutrición del 2018, presentación de resultados", s.f).

Según un estudio realizado por la Organización para la Cooperación y el Desarrollo Económicos (OCDE, 2017), se prevé que en el 2030 la obesidad aumentará en países como Estados Unidos de América en un porcentaje del $47 \%$, en México 39\% y en Inglaterra un 35\%, lo anterior resulta alarmante ya que del mismo modo que aumenta la obesidad aumentan sus consecuencias, entre las cuales destacan: enfermedad coronaria, diabetes, hipertensión, dislipidemia, accidente cerebrovascular, enfermedad del hígado o la vesícula, apnea del sueño o problemas respiratorios, artrosis, problemas ginecológicos, entre otros (Centros para el Control y Prevención de Enfermedades [CDC], 2020). Además, se han identificado problemas relacionados con la salud emocional, como autoestima baja y depresión, que se presentan comúnmente en niños (National Institutes of Health $[\mathrm{NIH}]$, s.f).

Aunado a lo anterior, es bien conocido que el riesgo de padecer alguna Enfermedad No Transmisible (ENT) en la edad adulta, como también tener dificultades respiratorias, riesgo de fracturas y algunos efectos psicológicos (OMS, 2020) como la insatisfacción corporal (Saldaña, 2005), suelen ser 
algunas de las consecuencias de la obesidad con mayor impacto en la salud. Algunos autores señalan que el hecho de "sentirse obeso" puede afectar la manera en que una persona se siente, se tiene una imagen pobre de sí mismo que se manifiesta en sentimientos de inferioridad y rechazo, conduce al aislamiento, inactividad, depresión y por ende se produce un aumento en la ingesta de alimentos (Mestas, Gordillo, Arana \& Salvador, 2012; Pallares y Baile, 2012).

La multicausalidad de la obesidad abarca tanto aspectos biológicos como psicosociales. Se considera que las personas con obesidad son psicológicamente diferentes a los demás, debido a que está asociada a la depresión, ansiedad y baja autoestima (Ortega, Fernández-Canet, ÁlvarezValdeita, Cassinello \& Baguena-Puigcerver, 2012). Sus principales factores de riesgo son la inactividad física, el sedentarismo, la ingesta de alimentos con alta densidad energética y en grandes porciones, el consumo de refrescos y bebidas azucaradas, y una frecuente ingesta de alimentos entre comidas (Rivera, et al. 2018).

Cabe destacar que así como existen factores que conllevan un riesgo en las personas para presentar obesidad, se encuentran los que promueven cambios favorables en las conductas de salud, entre ellos la autoeficacia, definida como la creencia de la propia capacidad para responder ante situaciones cotidianas, es decir, que tan capaz se siente el individuo de modificar su estilo de vida con el objetivo de controlar su peso (Aguiar, Magallanes, Martínez, Negrete \& García, 2017; Gómez-Acosta, 2018).

Bandura (1977), hace referencia a la autoeficacia como el conjunto de creencias del individuo sobre sus posibilidades de acometer y completar tareas con éxito, por lo anterior, la autoeficacia es fundamental para el 
control de peso de los adolescentes, y se le ha considerado como un factor de gran importancia en el mantenimiento de la salud, ya que contribuye en la mejora de los hábitos e influye directamente en la percepción del bienestar y la actitud positiva que se tiene ante el cambio de conductas saludables. Se ha demostrado en diversas investigaciones, que existe una asociación entre los bajos niveles de autoeficacia y la disminución de la capacidad de controlar el peso corporal, una menor adherencia a tratamientos, aumento de ansiedad y depresión (Aguiar, Magallanes, Martínez, Negrete \& García, 2017; Landero-Olvera, Guevara-Valtier, Vera-Sánchez, López-Valvarenga, \& Paz-Morales, 2017; Palacios \& Ramírez, 2016; Silva, Fernández, \& Rodríguez, 2019).

La evidencia empírica indica que hay relación entre el aumento de la autoeficacia y la mejora de estilos de vida saludables, el aumento en la actividad física en adolescentes, mayor cuidado de la salud y la percepción de una imagen positiva (Gaspar-Pérez, et al., 2019; Landero-Olvera, et al., 2017; Silva, et al., 2019). Cabe mencionar también, que se ha probado el efecto de un programa de autoeficacia para el control de peso en muestras de niños de la Ciudad de México, cuya evidencia muestra un incremento significativo en autoeficacia global y sus dimensiones: actividad física, disminuir consumo de alimentos con bajo aporte nutricional, hábitos saludables y alimentación emocional (Gómez- Peresmitré, Eguía-Platas, León, Pineda \& Guzmán, 2016; Gómez-Peresmitré, Platas-Acevedo y Pineda-García, 2019). En la ciudad de Pachuca Hidalgo, la versión de dicho programa disminuyó significativamente la autoatribución negativa en adolescentes (Gaspar-Pérez, et al., 2019).

Dala la importancia de la autoeficacia asociada al control de peso, realizar estudios que aporten conocimiento sobre las variables que predicen 
el incremento de autoeficacia será de suma importancia si se considera el grave problema de obesidad al que México se enfrenta. Entre dichos predictores puede tener lugar el afecto. Freedman, Kaplan \& Sadock (1975) mencionan que el afecto es considerado como la tonalidad sentimental vinculada a un objeto, idea o presentimiento, esto incluye sentimientos íntimos y sus manifestaciones externas (como se citó en Zamora, 2012).

Por su parte Watson, Clark y Tellegen (1988) señalan que el afecto positivo se caracteriza por elevada energía, motivación, deseos de afiliación, concentración, dedicación, sentimientos de dominio, logro y éxito. Los afectos positivos son considerados como un factor que puede prevenir enfermedades y facilitar un buen rendimiento. Lo contrario a los afectos negativos que se distinguen por la presencia de tristeza y letargo (como se citó en González, López \& Valdez, 2015), además se ha encontrado una asociación entre afecto positivo y autoestima (Crocker, Sommers \& Luhtanen, 2002).

Con base en la investigación documental, que evidencia el problema de obesidad, y en la importancia de identificar los factores que contribuyen al desarrollo de autoeficacia en la ámbito de la salud, el presente estudio tiene por objetivo identificar predictores de autoeficacia para el control de peso en estudiantes de secundaria (públicas y privadas) de la zona sur de Tamaulipas (Tampico y Ciudad Madero).

\section{METODOLOGÍA}

\section{Muestra}

La muestra no probabilística e intencional estuvo formada por 343 adolescentes estudiantes de dos escuelas secundarias públicas y dos privadas de Ciudad Madero y Tampico, Tamaulipas. El diseño de estudio fue explicativo y transversal. 


\section{Instrumentos}

La investigación implicó el uso de diversos instrumentos para medir las variables independientes y la dependiente:

El Inventario de Autoeficacia Percibida para el Control de Peso en su versión de adolescentes está integrado por 20 ítems. Su forma de calificación es obteniendo el valor promedio y su interpretación con base en el formato de respuesta 1) no soy capaz de hacerlo, 2) soy capaz de intentarlo, 3) soy capaz de hacerlo y 4) soy muy capaz de hacerlo. La validez de constructo se obtuvo mediante un análisis factorial exploratorio, un análisis factorial confirmatorio e invarianza factorial. Los indicadores de bondad de ajuste para el confirmatorio fueron: X2/g1 $\leq 3$, RMSEA $\leq$ $0.05 \mathrm{GFI} \geq 0.90, \mathrm{AGFI} \geq 0.90, \mathrm{CFI} \geq 0.95, \mathrm{NNFI} \geq 0.90$ y $\mathrm{SMRR} \leq 0.05$ y para la invarianza factorial: $\mathrm{X} 2>0.05, \mathrm{CFI} \leq 0.01$ y $\mathrm{RMSEA} \leq 0.015$. El instrumento final quedó integrado por 20 ítems que se agruparon en 2 factores: 1) Autoeficacia para la realización de actividad física y 2) Autoeficacia hacia la alimentación saludable. Se realizaron análisis por sexo: la versión de mujeres explicó $52 \%$ con 14 ítems y una alpha de Cronbach $=.90$. La versión de hombres explicó el $60 \%$ de la varianza con un alpha 0.90 (Gómez-Peresmitré, Platas, Pineda-García, GuzmánSaldaña, León-Hernández \& Calleja, 2018).

El índice de masa corporal (IMC) se obtuvo mediante el peso y la talla, siguiendo la siguiente fórmula ( $\mathrm{IMC}=$ peso/talla2). El peso se midió con una báscula digital marca mocrolife modelo WS100 con una precisión de $0.01 \mathrm{~kg}$ y un estadímetro de madera.

Escala de afecto positivo y negativo (PANAS) para niños y adolescentes. Está formada por 18 ítems con una escala de respuesta tipo Likert que va 
de "Nunca" (1) hasta "Siempre" (5). La confiabilidad se obtuvo mediante alfa de Cronbach $=0.82$. La validez de constructo se obtuvo mediante un análisis factorial exploratorio con método de componentes principales y rotación varimax. Se obtuvieron dos dimensiones que evalúan experiencias emocionales y explican el $45.2 \%$ de la varianza: 1) Afecto positivo con 8 ítems (alfa de Cronbach $=0.84$, varianza explicada $=19.9 \%$ ) y 2 ) afecto negativo con 10 ítems (alfa de Cronbach $=0.85$, varianza explicada $=$ 25.3\%) (Gómez-Peresmitré, Platas, Pineda, Corona \& González, 2018)

Inventario de Autoatribución que está integrado por 29 ítems con formato de respuesta tipo Likert que va desde "No me describe nada" (1) hasta "Me describe exactamente" (5). La confiabilidad para adolescentes mexicanos se obtuvo mediante alfa de $\mathrm{Cronbach}=0.83$. La validez se obtuvo mediante un análisis factorial exploratorio con método de componentes principales y rotación varimax. Está integrado por dos dimensiones que explican el 33\% de la varianza total: 1) autoatribución positiva con 17 ítems (alfa de Cronbach $=0.88$, varianza explicada $=20.25 \%$ ) y 2 ) autoatribución negativa con 12 ítems (alfa de Cronbach $=0.80$, varianza explicada $=$ 12.97\%) (Gómez-Peresmitré, Platas, Pineda, Rocha \& Guzmán, 2018)

\section{Procedimiento}

En primera instancia, un Doctor en Psicología colaborador del macroproyecto que lidera la Facultad de Psicología de la Universidad Nacional Autónoma de México, capacitó a 6 pasantes de la Licenciatura en Enfermería de la Universidad Autónoma de Tamaulipas Campus TampicoMadero, para estandarizar la aplicación de las medidas antropométricas y la batería de pruebas. Lo anterior con el fin de uniformar criterios para uniformar la recolección de datos y minimizar los errores. 
Posteriormente se contactó a los directivos de 2 escuelas secundarias privadas y una pública, para darles a conocer el contenido y objetivos del proyecto y solicitar la autorización para su implementación. Con previa autorización, se realizó una sesión informativa con padres de familia en la que se describió el objetivo y contenidos de dicho programa, así también se solicitó la firma del consentimiento para la participación de sus hijos, estudiantes menores de edad. Posteriormente se aplicaron los instrumentos, y se tomaron el peso y la talla a los escolares bajo su asentimiento y en presencia de un profesor de las instituciones educativas. Es importante señalar que este trabajo se apegó al código ético de psicología y a la ley general de salud en materia de investigación (Secretaría de salud, 2014).

\section{Análisis de información}

El procesamiento y análisis de datos se realizó mediante el Paquete Estadístico para las Ciencias Sociales (SPSS por sus siglas en inglés) versión 25 IBM para Windows (IBM Corp. 2007). La captura de datos la realizó una persona con dominio del paquete estadístico. Se realizó el análisis de datos perdidos u opciones de respuesta fuera del rango de numeración, los valores perdidos o datos atípicos se verificaron y corrigieron con base en los instrumentos en físico. Con el propósito de reducir sesgos en los análisis estadísticos, la base de datos fue revisada utilizando el $10 \%$ de los instrumentos en forma aleatoria para cotejar los datos capturados. Finalmente, para el análisis se utilizó estadística descriptiva mediante análisis de frecuencias, porcentajes, medidas de tendencia central y de dispersión. Para el análisis inferencial se aplicó un análisis de regresión lineal con el método de pasos. 


\section{RESULTADOS}

La edad de los participantes estuvo entre 11 y 17 años, con una media de 13.48 y desviación estándar de 1.03. Como se observa en la tabla 1, el porcentaje de mujeres y hombres fue similar (48.7 y 50.3\%), la mayoría de los estudiantes cursaba en una escuela secundaria pública (79\%), finalmente se observa que el porcentaje respecto al grado escolar fue equitativo $(34,30$ y $36 \%)$.

\section{Tabla 1.}

Distribución de variables sociodemográficas.

\section{Variable}

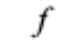

$\mathrm{n}=343$

167

176

Hombre

Tipo de escuela

Pública

Privada

Grado escolar

Primero

Segundo

Tercero
271

72

118

103

122
$\%$

$100 \%$

$48.7 \%$

$51.3 \%$

$79 \%$

$21 \%$

$34.4 \%$

$30 \%$

$35.6 \%$

Nota: $f=$ frecuencia, $\%=$ porcentaje.

\section{Fuente: Cedula sociodemográfica de los participantes.}

Para alcanzar el objetivo de estudio se aplicó un análisis de Regresión Lineal Múltiple con el método de pasos. La variable dependiente fue autoeficacia para el control de peso y las independientes: edad, IMC, autoatributos positivos, autoatributos negativos, afectos positivos y afectos negativos. El 
análisis concluyó en el paso 2, el ANOVA $(F=52.83, p<.001)$ indica que existe una relación lineal significativa entre dos variables independientes con la dependiente.

El modelo obtenido (ver figura 1) indica que la variable autoatributos positivos es la que mejor predice autoeficacia para el control de peso $(\beta=.38)$, seguida por afectos positivos $(\beta=.15)$. La varianza explicada para la dependiente es de 23\% y el estadístico Durbin-Watson (2.104) indica que existe independencia en los residuos. Otro aspecto relevante del modelo fue que se excluyeron las variables edad, IMC, autoatributos negativos y afectos negativos.

Figura 1. Modelo de predictores de autoeficacia para el control de peso.

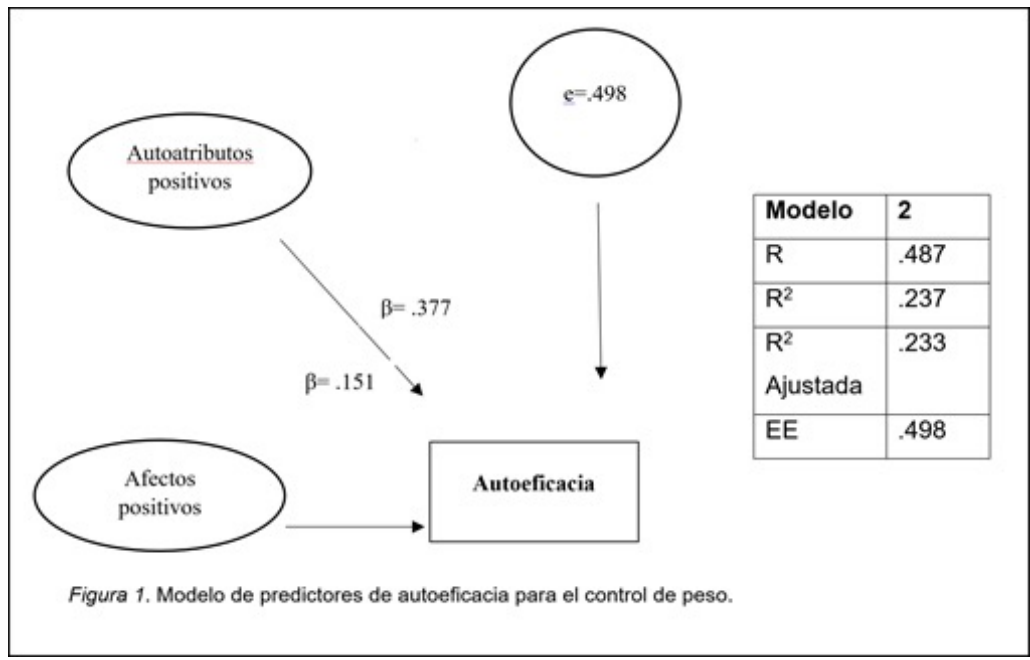

\section{DISCUSIÓN Y CONCLUSIONES}

A partir de los resultados, se puede concluir que el modelo predictivo de autoeficacia para el control de peso en estudiantes adolescentes de la zona sur de Tamaulipas es significativo y cumple con el objetivo de este 
estudio que fue identificar predictores de autoeficacia para el control de peso en estudiantes de secundaria (públicas y privadas) de la zona sur de Tamaulipas (Tampico y Ciudad Madero).

En primera instancia se demostró que el sexo que mayor predomino en los estudiantes es el masculino con un 51.3\%, dicho resultado no concuerda con estudios antecedentes, donde se incluyeron mayores porcentajes de mujeres (entre 70\% y 100\%) (Aguiar, et al. 2017; Silva, et al. 2019). Respecto al grado escolar, los estudiantes cursaban en una escuela secundaria publica con un $79 \%$, lo cual concuerda con el estudio de Gaspar-Pérez, et al. (2019).

Los análisis del modelo de regresión muestran que la variable dependiente autoeficacia se puede predecir a partir de dos variables independientes: la variable con mayor importancia resultó ser autoatributos positivos, seguida por afectos positivos. Dicho modelo reviste interés por diferentes aspectos. El primero de ellos radica en que no se detectaron estudios que identifiquen predictores de autoeficacia para el control de peso en la revisión de literatura especializada, por tanto, los resultados contribuyen a mejorar el conocimiento entre las interacciones causa efecto en las variables de estudio.

El segundo aspecto es que existe evidencia que apoya que tanto la autoeficacia, como su predictor afecto positivo, son fundamentales para el control de peso, en la percepción de imagen corporal positiva, bienestar, prevención de enfermedades y en el mantenimiento de la salud física (ejercicio y estilos de vida saludables) y mental (disminuye ansiedad y depresión) (Aguiar et. al., 2017; Gaspar-Pérez, et al., 2019; González, et al. 2015; Landero-Olvera, et al. 2017; Palacios \& Ramírez, 2016; Silva et. al., 2019). 
Otro aspecto de interés es que el presente modelo contribuye al avance de conocimiento en un tema que representa un problema de salud pública como la obesidad, que ha sido ampliamente estudiado a nivel mundial y en México, mostrando altas prevalencias, graves consecuencias y no muy prometedoras estimaciones a futuro (CDC 2020; Encuesta Nacional de Salud y Nutrición del 2018; Mestas, et al. 2012; OCDE, 2017; OMS, 2020; NIH, s.f; Pallares \& Baile, 2012; Saldaña, 2005).

Algunos otros aspectos de importancia en el modelo fue que se excluyeron variables consideradas en el análisis (edad, IMC, autoatributos negativos y afectos negativos) y que el modelo explica aproximadamente un cuarto de la varianza (deja de explicar el 77\%). Lo anterior, en lugar de representar una desventaja, es un elemento a considerar para futuras investigaciones en las que se exploren más variables que predicen autoeficacia para el control de peso, que sin bien es cierto no se encontró literatura al respecto, si es una oportunidad para entender la complejidad de un problema de salud como la obesidad y su multicausalidad identificada en diferentes investigaciones (Ortega et. al., 2012; Rivera et. al., 2018).

Ahora bien, entre las limitantes de este estudio se encuentra que fue un muestreo no probabilístico, por tanto no se pueden hacer inferencias a nivel poblacional, por lo tanto se sugiere realizar muestreos probabilísticos en distintas regiones del país, con diferentes grupos etarios y estratificando por categorías de IMC. Así también se sugiere incluir otras variables en los estudios, con la finalidad de documentar su influencia en la autoeficacia para el control de peso, lo anterior intentado incrementar el porcentaje de varianza explicado en el presente estudio. Finalmente se sugiere realizar estudios longitudinales que permitan realizar afirmaciones contundentes sobre las relaciones causa efecto entre las variables de este estudio. 
Finalmente, se concluye que el modelo aporta sustento empírico para diseñar intervenciones para el control de peso, en las que se consideren los temas de autoeficacia, autoatributos positivos y afectos positivos, que han demostrado con anterioridad, resultados positivos en intervenciones con muestras de niños y adolescentes mexicanos (Gaspar-Pérez, et al., 2019; Gómez- Peresmitré, et al., 2016; Gómez-Peresmitré, 2019).

\section{REFERENCIAS}

Aguiar, P. L., Magallanes, R. A., Martínez, A. J., Negrete, C. A., \& García, G. M. (2017). Autoeficacia para el control de peso en estudiantes universitarios del área de la salud. Revista Latinoamericana de Ciencia Psicológica, 9(1). doi: http://dx.doi.org/10.5872/psiencia.v9i1.212

American Psychological Association. (s.f). Salud mental/ corporal: La obesidad. Recuperado de https://www.apa.org/centrodeapoyo/ obesidad

Bandura, A. (1997). "Self-efficacy: Toward a unifying theory of behavioral change". Psychological rewiew, 84(1), 19-215.

Centros para el Control y Prevención de Enfermedades. (2020). Los efectos del sobrepeso y la obesidad en la salud. Nota descriptiva. [Internet]. Recuperado de https:/www.cdc.gov/healthyweight/ spanish/effects. html

Crocker, J., Sommers, S. R., \& Luhtanen, R. K. (2002). Hopes Dashed and Dreams Fulfilled: Contingencies of Self-Worth and Graduate School Admissions. Personality and social psychology bulletin, 28(9), 12751286. doi: https://doi.org/10.1177/01461672022812012

Dávila-Torres, J., Gonzalez-Izquierdo, J., y Barrera-Cruz, A. (2015). Panorama de la obesidad en México. Revista Médica del Instituto Mexicano del Seguro Social, 53(2), 241-209. Recuperado de http:// revistamedica.imss.gob.mx/editorial/index.php/revista_medica/ article/viewFile/21/54 
Encuesta Nacional de Salud y Nutrición 2018. (s.f). Presentación de resultados. Recuperado de https://www.inegi.org.mx/programas/ ensanut/2018/

Gaspar-Pérez, A., Guzmán-Saldaña, R., Bosques-Brugada, L., PlatasAcevedo, S., Vázquez-Arévalo, R., \& Guadarrama-Guadarrama, R. (2019). Programa en autoeficacia, afecto positivo y autoatribución para promover conductas alimentarias saludables en estudiantes de secundaria. Salud y Educación, 8(15), 24-30. doi: https://doi. org/10.29057/icsa.v8i15.4913

Gómez, S. F. (2016). Desnutrición. Boletín médico del hospital infantil de México, 73(5), 297-301. doi: 10.1016/j.bmhimx.2016.07.002

Gómez-Acosta, C. (2018). Factores psicológicos predictores de estilos de vida saludable. Revista de Salud Pública, 20(2). doi: 10.15446/rsap. v20n2.50676

Gómez-Peresmitré, G., Eguía-Platas, A. S., León, H. R., Pineda, G. G., \& Guzmán, S. R. (2016). Resultados preliminares de un programa de prevención de obesidad basado en la autoeficacia para control de peso. Ciudad de México, México: Asociación Mexicana de Psicología Social A.C.

Gómez-Peresmitré, G., Platas-Acevedo, S., \& Pineda-García, G. (2019). Programa de autoeficacia hacia hábitos saludables para la prevención de la obesidad en escolares mexicanos. Psicología clínica con niños y adolescentes, 6(1), 44-50. doi: 10.21134/rpcna.2019.06.1.6

Gómez-Peresmitré, G., Platas, A. S., Pineda, G.G., Corona, L.N., y González, B. V. (2018). Propiedades psicométricas de la escala de afecto negativo y afecto positivo (PANAS) en adolescentes de secundaria. La psicología social en México. 17(1), 463-475.

Gómez-Peresmitré, G., Platas, A. S., Pineda, G.G., Rocha, S. P., y Guzmán, S.R. (2018). Propiedades psicométricas del inventario de autoatribución para adolescentes de secundaria. La psicología social en México, 17(1), 361-374.

Gómez-Peresmitré, G., Platas, A. S., Pineda, G., Guzmán, S., León, H., y Calleja, N. (2021). Multi-validity proces and factor- invariance. 
Perceived self-efficacy-scale for the prevention of obesity in preadolescents. Children, 8, 504. doi: https://doi.org/10.3390/ children8060504

González, N., López, F. A., \& Valdez, M. J. (2015). Validez de las escalas de afecto positivo y negativo (PANAS) en niños. Liberabit, 21(1), 37-47. Recuperado de http://www.scielo.org.pe/scielo.php?script=sci _arttext\&pid=S1729-48272015000100004\&lng=es\&tlng=es

Gutiérrez, V. J., Guevara, V. M., Enríquez, R. M., Morales, P. M., de los Ángeles, P. M., del Ángel, H. M., \& Landeros, O. E. (2017). Estudio en familias: factores ambientales y culturales asociados al sobrepeso y obesidad. Enfermería Global, 16(45), 1-19. doi: http://dx.doi. org/10.6018/eglobal.16. 1.25381121440009

IBM Corp. (2007). SPSS for Windows, Version 25.0. Chicago, IL: SPSS Inc. [Computer sofware].

Landero-Olvera, E. A., Guevara-Valtier, M. C., Vera-Sánchez, I., LópezAlvarenga, J. C., \& Paz-Morales, M. A. (2017). Impacto de un programa de ejercicio sobre la Autoeficacia en mujeres con sobrepeso y obesidad. Revista de Medicina e Investigación, 5(1), 47-53. Recuperado de http://hdl.handle. net/20.500.11799/66656

Mestas, L., Gordillo, F., Arana, J. M., \& Salvador, J. (2012). Síntesis y nuevas aportaciones para el estudio de la obesidad. Revista Mexicana de Trastornos Alimentarios, 3(2), 99-105. Recuperado de http://www. scielo. org.mx/pdf/rmta/v3n2/v3n2a3.pdf

National Institutes of Health. (s.f.). Sobrepeso y obesidad. Recuperado de https://www.nhlbi.nih. gov/health-topics/espanol/sobrepeso-yobesidad

Organisation for Economic Cooperation and Development. (2017). Obesity Update. Recuperado de http://fmdiabetes.org/la-ocde-presento-elinforme-de-actualizacion-sobre-la-obesidad-2017/

Organización Mundial de la Salud. (2018). Malnutrición. Nota descriptiva. [Internet]. Disponible en https://www.who.int/es/news-room/factsheets/detail/malnutrition 
Organización Mundial de la Salud. (2020). Obesidad y sobrepeso. Nota descriptiva. [Internet]. Disponible en https://www.who.int/es/newsroom/fact-sheets/detail/obesity-and-overweight

Ortega, J., Fernández-Canet, R., Álvarez-Valdeita, S., Cassinello, N., \& Baguena-Puigcerver, M. J. (2012). Predictors of psychological symptoms in morbidly obese patients after gastric bypass sugery. Surgery for Obesity and Related Diseases: Official Journal of the American Society for Bariatric Surgery, 8(6), 770-776. doi: 10.1016/j. soard.2011.03.015.

Pallares, J., \& Baile, J. (2012). Estudio de la obesidad infantil y su relación con la imagen corporal en una muestra española. Psychologia: Avances de la Disciplina, 6(2), 13-21. Recuperado de https://dialnet.unirioja. es/servlet/articulo? codigo $=4924012$

Saldaña, G. C. (2005). Repercusiones psicológicas de la obesidad en la adolescencia. FMC - Formación Médica Continuada En Atención Primaria, 12(3), 139-146. doi:10.1016/s1134-2072(05)71184-1

Secretaría de Salud. (2014). Reglamento de la ley general de salud en materia de investigación para la salud. Recuperado de http://www.salud.gob. $\mathrm{mx} /$ unidades/cdi/nom/compi/rlgsmis.html

Silva, C., Fernández, N., \& Rodríguez, N. (2019). Depresión, ansiedad $\mathrm{y}$ autoeficacia para bajar de peso en mujeres en tratamiento nutricional. Psicología y Salud, 29(1), 41-49. Recuperado de http:// psicologiaysalud. uv.mx/index.php/psicysalud/article/view/2567

Rivera, D. J., Colchero, M. A., Fuentes, M. L., González, C. T., Aguilar, S. C., Hernández, L. G., \& Barquera, S. (2018). La obesidad en México. Estado de la política pública y recomendaciones para su prevención y control. Recuperado de https://www.insp.mx/avisos/4884-laobesidad-mexico.html

Zamora, C. M. (2012). La sensibilidad a la ansiedad, afectos positivosnegativos, evitación agorafóbica y su relación con el hábito de fumar incipiente (Tesis de maestría). Recuperada de http://132.248.9. 195/ ptd2013/Presenciales/0702283/Index. html 
Envío a dictamen: 17 de junio de 2021

Reenvío: 12 de agosto de 2021

Aprobación: 27 de agosto de 2021

Dr. Rodrigo César León Hernández. Investigador por México CONACYT. Miembro Del Sistema Nacional De Investigadores Nivel I. Licenciatura y el Doctorado en la UNAM. Especialidad: en el Tratamiento De Los Trastornos De La Conducta Alimentaria: Anorexia Y Bulimia Nerviosa. Hospital Clínic De Barcelona. Docente en Distintas Instituciones De Educación Superior: EED-ISSSTE, UNAM, UVM, UJS, FET-UAT Y UNE.

Participación Activa Como Colaborador O Responsable En Proyectos De Investigación Financiados Por EL CONACYT, PAPIIT, IMJ, INMUJERES, SS, SEP, ONU, MAPFRE, PFIZER. INEN-PERÚ. UV. Autor O Coautor En Publicaciones De Divulgación Científica (Memorias, Artículos, Capítulos De Libros Y Libros).

Dra. Gilda Gómez Peremitré. Profesora Titular "C" T. C. de la Facultad de Psicología en la UNAM. Obtuvo un MD en The University of Texas at Austin, y una Especialización en la Universidad de Jerusalén, en Israel. Es pionera en la investigación en Factores de Riesgo en Trastornos de la Conducta Alimentaria, obesidad e imagen corporal en México, así como en el estudio de los trastornos alimentarios en hombres y orientación sexual. Autora de un instrumento online para la detección de factores de riesgo para los TCA Colabora con investigadores de la Universidad de Hannover, Alemania; el Instituto clínico de Psiquiatría y Psicología de Barcelona, y con la Universidad de Almería en España.. Es miembro del Sistema a Nacional de Investigadores nivel II. 
Gregorio Méndez Santos. Graduado de la Lic. en Enfermería de la Universidad Autónoma de Tamaulipas (UAT), Miembro activo y Ex Subjefe de la Oficina Nacional de Documentación en la Asociación Mexicana de Estudiantes de Enfermería (AMEENF), coautor de artículos científicos y colaborador de proyectos de investigación en el área de la salud. Actualmente becario de la fundación Pineda World Enable en Berkeley, CA, EUA.

Yulissa Guadaupe Torres Compeán. Licenciatura en la Universidad Autónoma de Tamaulipas, Miembro activo de la Asociación Mexicana de Estudiantes de Enfermería, Representante Estatal de la Oficina de Investigación en Tamaulipas de la AMEENF, autora y coautora de Publicaciones de Divulgación Científica. 